\title{
ANNULAR PANCREAS IN THE NEWBORN
}

BY

\author{
P. P. RICKHAM \\ From Alder Hey Children's Hospital, Liverpool
}

(RECEIVED FOR PUBLICATION AUGUST 26, 1953)

In annular pancreas a collar or ring of tissue surrounds the second part of the duodenum. It is a rare malformation. Vidal published the first case in 1905; his patient was a newborn baby. In a recent publication, Swynnerton and Tanner (1953) collected 76 cases and we have found one more in the literature (MacPhee, 1953). In 1933 McNaught could only find 40 instances of this condition in the literature, most of them necropsy findings, but during the last three years over 20 cases have been published, all of them discovered at operation.

As annular pancreas is a congenital malformation, it is surprising that only nine of the 77 recorded cases were found in newborn babies. It therefore seems worth recording that we have encountered

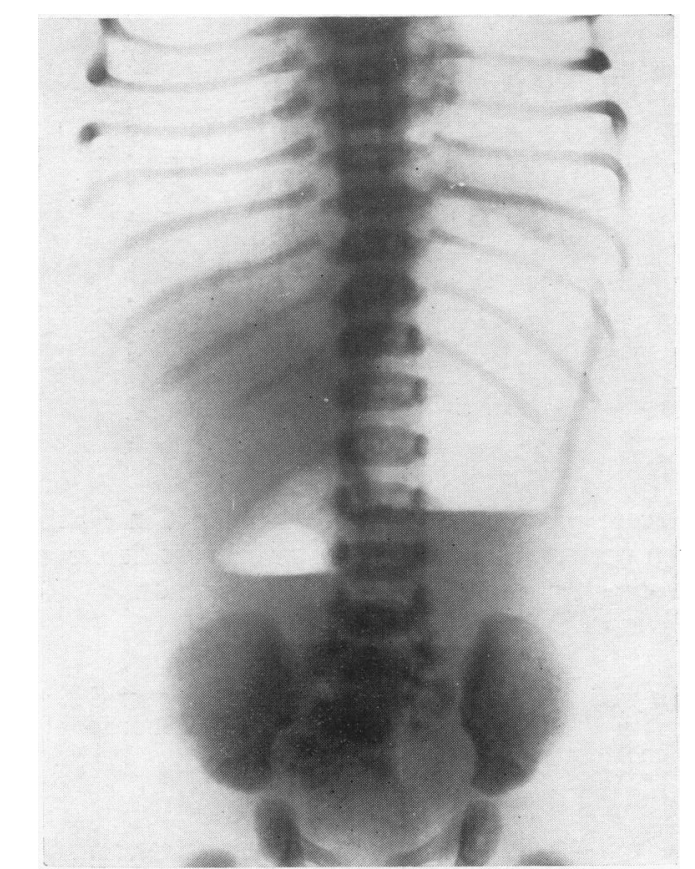

FIG. 1.-Case 1: radiograph of abdomen (patient sitting up). There are large air bubbles in the stomach and first part of the duodenum, but no air in the rest of the intestine. five neonates with this malformation during the last 18 months. It is likely that in the past many of the babies with annular pancreas giving rise to obstructive symptoms have died unoperated upon and undiagnosed. With the rapid advance of neonatal surgery, it is safe to predict that many more will be discovered and treated in the future.

\section{Case Reports}

The following are reports of three cases presenting with duodenal obstruction during the first few days of life:

Case 1.-Stephen was admitted on November 3, 1951, aged 2 days. He had been deeply cyanosed since birth and had vomited bile-stained material.

On examination, in addition to cyanosis, there was gaseous distension of the epigastric region and visible gastric peristalsis.

An x-ray film confirmed the diagnosis of duodenal obstruction (Fig. 1).

At operation the second part of the duodenum was found to be tightly constricted by a ring of pancreatic tissue. A duodenojejunostomy was performed.

Recovery was uneventful. The cyanosis persisted and was later found to be due to tricuspid atresia. M r. R o n a ld Edwards performed a thoracotomy when the boy was 12 months old, but decided not to

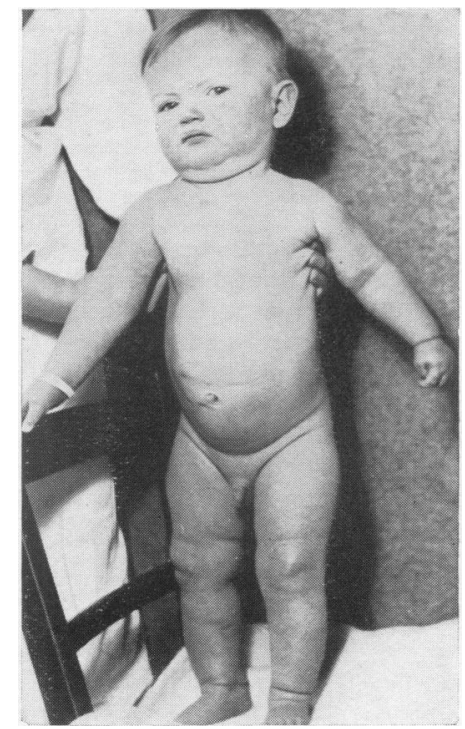

FIG. 2.-Case 1 : the patient 18 months after operation. undertake corrective measures. In spite of severe cyanosis, the child continues in good health (Fig. 2).

Case 2.-Ronald was admitted on November 17, 1952, when 7 days old. He was a premature baby weighing 


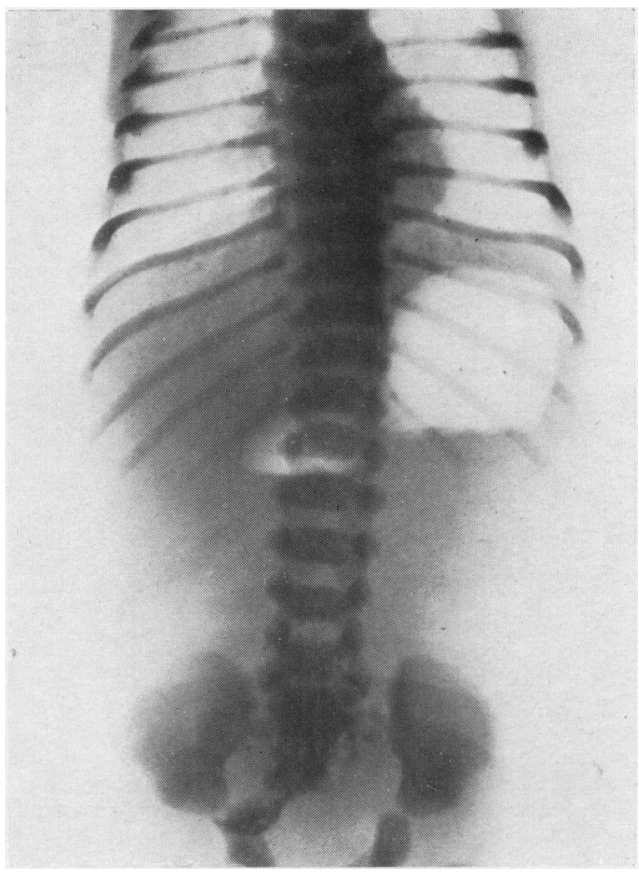

FIG. 3.-Case 2: radiograph of the abdomen (patient sitting up). The appearance is similar to Fig. 1 .

$2 \frac{1}{2} \mathrm{lb}$. He had vomited bile-stained material for four days.

On examination he was severely dehydrated and had signs of bilateral pneumonia. A diagnosis of duodenal

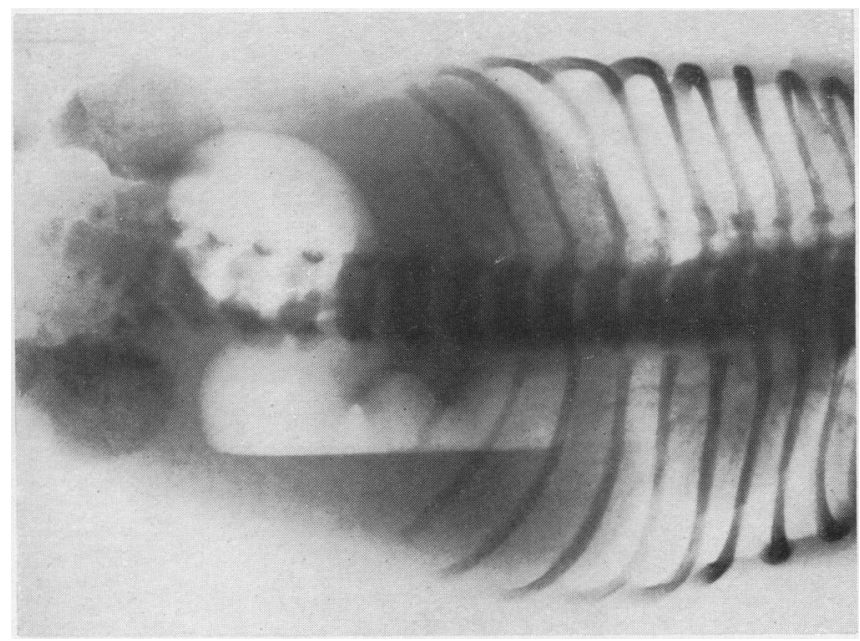

Fig. 4.-Case 3: radiograph of the abdomen (patient lying on the left side). The stomach and first part of the duodenum are outlined by air. There is no air in the rest of the small intestine. obstruction was made on the x-ray films (Fig. 3). Intravenous infusion and gastric suction were instituted for 24 hours before operation.

At operation, there was gross dilatation of the proximal duodenum. Owing to the child's precarious condition, no attempt was made to dissect out the duodenum, which was considered to be atresic. A duodeno-jejunostomy was performed.

The baby appeared to be fairly well post-operatively but died a week later of pneumonia and prematurity.

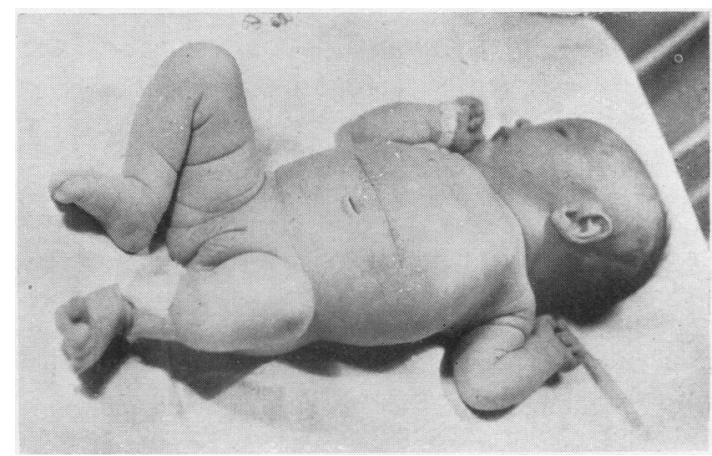

FIG. 5.-Case 3: the patient two months after operation.

Necropsy showed, in addition to bilateral pneumonia, a short segment of duodenal atresia at the level of the ampulla of Vater. The duodenum was surrounded by a ring of pancreatic tissue.

Case 3.-Betty was admitted on January 10, 1953, aged 3 days, with a history of vomiting bile-stained material since birth.

A diagnosis of duodenal obstruction was made on the history, examination and $x$-ray findings (Fig. 4).

At operation the second part of the duodenum was found to be tightly encircled by an annular pancreas. The enormously dilated proximal and collapsed distal duodenum were dissected out and a duodeno-duodenostomy was performed around the constricting ring of the pancreas. The baby made a satisfactory recovery (Fig. 5).

In two further neonates an annular pancreas was found at necropsy as an incidental finding. In both cases there were no symptoms of duodenal obstruction.

Case 4.- James was admitted on February 26,1953 , aged $3 \frac{1}{2}$ weeks, with bronchopneumonia; he died a few hours later.

At necropsy, an annular fancreas, 
without duodenal constriction, was discovered (Figs. 6 and 7). Enquiries established the fact that the child had had no symptoms of duodenal obstruction.

Case 5.-David was admitted on March 3, 1953. He had an oesophagotracheal fistula for which an end-to-end anastomosis was performed at the age of 24 hours.

He died of pneumonia one week later, having taken milk feeds and passed milk stools. At necropsy, a ring of pancreas was found surrounding the second part of the duodenum but not constricting it.

Aetiology.-The pancreas of birds is normally annular. The condition has been described in a $16 \mathrm{~mm}$. embryo by Weissberg (1935). The most commonly accepted explanation of this anomaly is persistence of the left half of the central pancreatic anlage.

Signs and Symptoms.- The severity of symptoms and the age at which they occur is presumably determined by the degree of constriction of the duodenum caused by the encircling pancreatic tissue. The adult group comes to surgery for chronic duodenal obstruction, peptic ulceration, or pancreatitis. Presumably some secondary change occurs before the congenital malformation causes symptoms, the degree of obstruction being slight. One might be allowed to speculate that if our two babies, in whom annular pancreas was an incidental

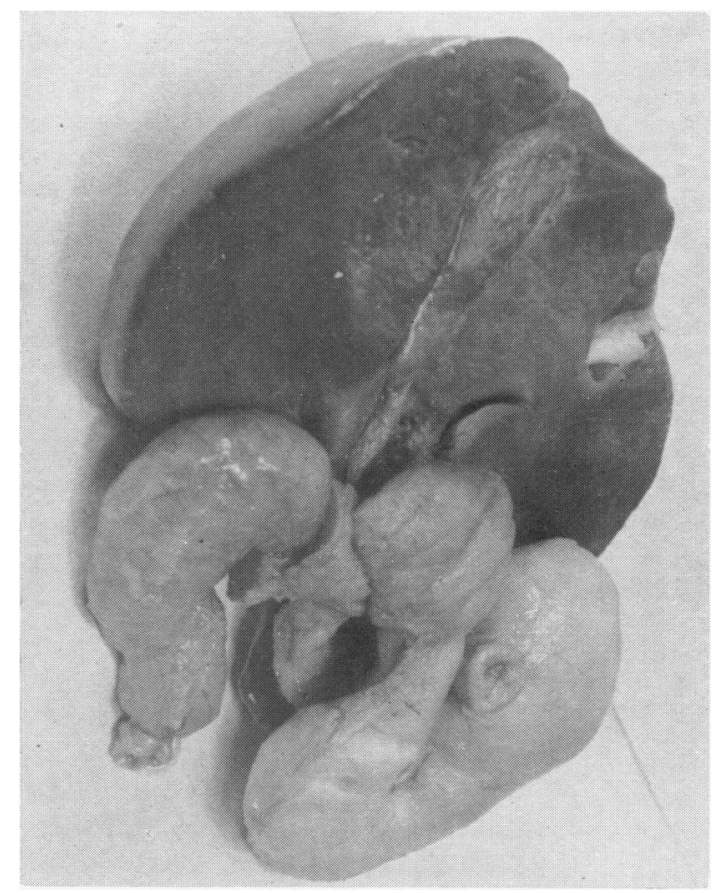

FIG. 6.-Case 4: post-mortem specimen showing the head of the pancreas surrounding the second part of the duodenum. finding, had not died, they too might have developed symptoms in later life. The infant group present with complete or incomplete duodenal obstruction at, or soon after birth, and the constriction of the duodenum is severe. Occasionally, obstructive

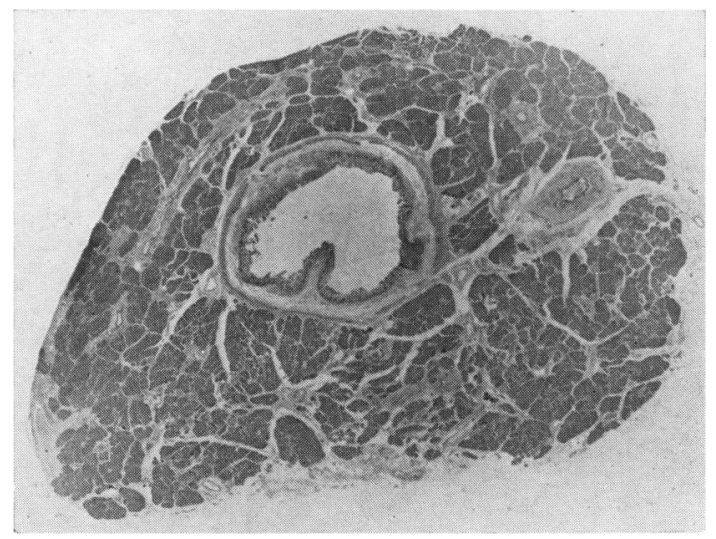

FIG. 7.-Case 4: horizontal section through the second part of the duodenum. The lumen of the gut is completely surrounded by pancreas. The common bile duct lies medial to the duodenum. The ampulla of one of the pancreatic ducts projects anteriorly into the lumen of the duodenum.

jaundice is present in addition to duodenal obstruction. This has been rather unsatisfactorily explained as being due to pressure on the common duct or ampulla of Vater.

Diagnosis. - In infants, the x-ray films confirm the diagnosis of duodenal obstruction. In none of our three cases had air passed beyond the second part of the duodenum. The films give no clue to the cause of the obstruction and a diagnosis of duodenal atresia is invariably made. Annular pancreas was diagnosed by Lehman (1942) in an adult on the radiographic appearance following a barium swallow. This is the only correct pre-operative diagnosis recorded in the literature. At operation, the diagnosis may be missed in an infant unless the proximal duodenum is dissected free (Case 2). It is perhaps worth noting that none of our 5 infants were mongols, for, in our experience, duodenal atresia is associated with a $50 \%$ incidence of mongolism.

Treatment.-In the adult group where obstructive symptoms are less marked, a wedge resection of the encircling ring of pancreatic tissue has been used as an alternative to a short-circuiting procedure. Such a resection would be insufficient in newborn babies as it would not cure the underlying duodenal stenosis or atresia. Duodeno-duodenostomy around the constricting ring of pancreas, if practical, would appear to be the ideal treatment. Failing this, 
duodeno-jejunostomy should be satisfactory. Gastrojejunostomy should be avoided; it will fail to drain the dilated proximal duodenum and incomplete obstruction will result in bile passing backwards into the stomach and the setting up of acute gastritis.*

* Since this paper was prepared we have operated on a further case of annular pancreas in the newborn. This child, however, was a mongol.
I should like to thank Miss I. Forshall for her help with the treatment of these infants and the preparation of this paper.

\section{REFERENCES}

Lehman, E. P. (1942). Ann. Surg., 115, 574.

MacPhee, I. W. (1953). Brit. J. Surg., 40, 510

McNaught, J. B. (1933). Amer. J. med. Sci., 185, 249.

Swynnerton, B. F. and Tanner, N. C. (1953). Brit. med. J., 1, 1028.

Vidal, E. (1905). 18è Congrès de Chirurgie, Paris, 1905. Procès verbaux, mémoires et discussion (Assoc. frans. Chir.), 18, 739.

Weissberg, H. (1935). Anat. Anz., 79, 296. 